\title{
Campylobacter jejuni invade chicken LMH cells inefficiently and stimulate differential expression of the chicken CXCLi1 and CXCLi2 cytokines
}

\begin{abstract}
Correspondence
Michael E. Konkel

konkel@wsu.edu
\end{abstract}

Received 9 June 2008

Revised 18 August 2008

Accepted 28 August 2008

\author{
Charles L. Larson, ${ }^{1}$ Devendra H. Shah, ${ }^{2}$ A. Singh Dhillon, ${ }^{3}$ \\ Douglas R. Call, ${ }^{2}$ Soohyoun Ahn, ${ }^{1}$ Gary J. Haldorson, ${ }^{4}$ Chris Davitt ${ }^{5}$ \\ and Michael E. Konkel ${ }^{1}$
}
${ }^{1}$ School of Molecular Biosciences, Washington State University, Pullman, WA 99164-4234, USA
${ }^{2}$ Department of Veterinary Microbiology and Pathology, Washington State University Pullman, WA 99164-7040, USA
${ }^{3}$ Washington State University Avian Health and Food Safety Laboratory, College of Veterinary Medicine, Washington State University, Puyallup, WA 98371-4998, USA
${ }^{4}$ Department of Veterinary Microbiology and Pathology and Washington Animal Disease Diagnostic Laboratory, Washington State University Pullman, WA 99164-7040, USA
${ }^{5}$ Franceschi Microscopy and Imaging Center, Washington State University, Pullman, WA 99164- 4236, USA

Campylobacter jejuni is a major food-borne bacterial pathogen, which is capable of causing diarrhoea containing blood and leukocytes. C. jejuni invasion of the intestinal epithelial cells and the release of proinflammatory molecules contribute to the pathophysiology of campylobacteriosis. Given the commensal relationship of C. jejuni with chickens, we hypothesized that C. jejuni invasion of chicken cells and the release of host cell cytokines would be significantly less than with human cells. To test our hypothesis, we examined the interactions of C. jejuni with chicken LMH cells, and performed in vivo experiments with chickens. The binding and internalization assays revealed that C. jejuni was significantly less invasive of LMH cells relative to human INT 407 cells, even though the bacteria bound to each host cell species equally. We also assessed interleukin-8 (IL-8) transcript, IL-8 secretion, and the release of chemoattractant molecules from the inoculated cells. Inoculation of $\mathrm{LMH}$ cells with $\mathrm{C}$. jejuni stimulated expression of both chicken IL-8 orthologues, chCXCLi2 and chCXCLi1, but at levels significantly less than human IL-8 (huCXCL8) expressed from human INT 407 cells inoculated with C. jejuni. Moreover, the supernatant fluids of the C. jejuni-inoculated LMH cells resulted in little heterophil migration. In vivo, C. jejuni were observed bound to the cells lining the glandular crypts, but overt signs of cell invasion or pathology were not observed. These results indicate that cytokine expression in chicken LMH cells in response to C. jejuni is distinct from that of Salmonella typhimurium.

\section{INTRODUCTION}

Campylobacter jejuni, a Gram-negative, spiral-shaped bacterium, is a common cause of gastrointestinal illness throughout the world. In developing countries, individuals are continuously exposed to C. jejuni from an early age. As a result of repeated exposure, $C$. jejuni-infected individuals can be asymptomatic or suffer from mild diarrhoea characterized by loose, watery stools (Blaser et al., 1985;

Abbreviations: $C_{t}$, cycle threshold; IL-8, interleukin-8; p.i., post-inoculation; PMA/I, phorbol 12-myristate 13-acetate and ionomycin; TEM, transmission electron microscopy.
Wassenaar \& Blaser, 1999). In contrast, C. jejuni-infected individuals in developed countries experience fever, abdominal cramps and diarrhoea that may be accompanied with gross blood and leukocytes (Black et al., 1988; Blaser et al., 1983). This observed variation in disease severity is likely to be due to differences in both bacterial virulence (i.e. host cell invasion) and the host innate immune response.

A complex set of events is necessary for a robust host inflammatory response (Kelly et al., 2005; Nathan, 2006). In one instance, pathogen-associated molecular patterns (PAMPs) are recognized by host pattern recognition 
receptors (PRRs) on the surface of intestinal epithelial cells (O'Hara \& Shanahan, 2006). This interaction triggers host cell signal transduction pathways and the transcription of genes encoding proinflammatory molecules. For example, Toll-like receptor 5 (TLR5) is found on the apical surface of intestinal epithelial cells. TLR5 recognizes conserved epitopes within some bacterial flagellins and initiates signalling that leads to the expression of the gene encoding interleukin-8 (IL-8, also known as huCXCL8) (Ramos et al., 2004). The release of IL-8 and other proinflammatory molecules from cells activates innate immune defences (i.e. inflammation) and promotes acquired immune responses. Other evidence suggests that PRR-dependent expression of proinflammatory molecules alone is not always sufficient to initiate the inflammatory response, but that the release of a second class of molecules from injured host cells may be required for robust inflammation (see reviews by Blikslager et al., 2007; Nathan, 2006). The host microflora can also influence the innate immune response. Specifically, bacteria can dampen the immune response and block the release of proinflammatory molecules in order to colonize a host. A commensal strain of Salmonella, which harbours PAMPs, blocks NF- $\kappa$ B-dependent expression of proinflammatory cytokines to prevent host inflammation (Neish et al., 2000).

A hallmark of gastrointestinal infection with $C$. jejuni is an acute host inflammatory response (Allos, 2001). Central to this acute inflammatory response is the secretion of IL-8 from the cells lining the intestinal tract (Wassenaar \& Blaser, 1999). IL-8 is a potent chemoattractant and activator of macrophages and polymorphonuclear leukocytes (Fleckenstein \& Kopecko, 2001). In humans, serum IL-8 levels peak between 3 and 10 days after the onset of the disease (Baqar et al., 2001), and it is common to observe gross blood and leukocytes in stool samples from these individuals (Konkel et al., 2001). The characteristics of clinical disease support the notion that proinflammatory mediators and tissue damage both participate in the robust inflammatory response (Baqar et al., 2001; Black et al., 1988). In vitro studies have revealed that $C$. jejuni invades and stimulates the release of IL-8 from numerous human colonic cell lines, and that the level of IL-8 released is related to the invasiveness of the $C$. jejuni strain and the host cell lineage (Bakhiet et al., 2004; Everest et al., 1992; Hickey et al., 1999; MacCallum et al., 2006).

C. jejuni infections frequently result from eating undercooked poultry or foods cross-contaminated with raw poultry products. C. jejuni is a commensal organism in chickens. Indeed, the caeca of chickens can be colonized by in excess of $10^{7}$ C. jejuni per gram of caecal contents without signs of pathology (e.g. host cell invasion, cell necrosis, inflammation, influx of neutrophils) (Beery et al., 1988; Cawthraw et al., 1994). The characteristics that distinguish the commensal relationship of $C$. jejuni with chickens from its pathogenic relationship with humans are unclear.
In vitro cell culture models have been employed to explore the interaction between $C$. jejuni and chicken epithelial cells. Specifically, studies have been performed to determine whether $C$. jejuni invades primary chicken epithelial cells and induces the expression of proinflammatory cytokines.

It has been reported that $C$. jejuni invades human epithelial cells and primary chicken cells at similar levels (Byrne et al., 2007; Smith et al., 2005). The array of proinflammatory cytokines produced by humans and chickens is distinct. Human IL-8 is expressed from the CXCL8 gene, for which chickens have two orthologues, termed CXCLi1 (K60) and CXCLi2 (CAF/IL-8, also described as 9E3/CEF4) (Kaiser et al., 1999; Sick et al., 2000). As with CXCL8 gene expression in human epithelial cells, researchers have observed an increase in CXCLi1 and CXCLi2 expression in primary chicken cells inoculated with C. jejuni (Borrmann et al., 2007; Smith et al., 2005). Based on the results from these studies, investigators have concluded that the interaction of C. jejuni with human cells is similar to that observed with chicken cells. We undertook this study with a continuous chicken epithelial cell line (LMH) as an in vitro model to further dissect the interactions of C. jejuni with chicken cells. To our knowledge, this is the first study to evaluate the use of LMH cells as an in vitro model to investigate the interaction of $C$. jejuni with chicken epithelial cells.

In this study, we report differences in invasiveness, expression and secretion of IL-8, as well as the functional activity of chemoattractants between chicken and human cells inoculated with $C$. jejuni. Throughout this manuscript, we refer to IL-8 from humans as huCXCL8 and IL-8 from chickens as chIL-8. In instances where they can be distinguished, we will refer to the specific CXCL8 orthologues as chicken CXCLi1 (chCXCLi1) and chicken CXCLi2 (chCXCLi2). We tested whether the inoculation of chicken LMH cells with $C$. jejuni induces these cells to secrete IL-8 and other chemoattractant molecules. In addition, chickens were inoculated with $C$. jejuni to assess colonization, the immune response, and the pathology of the caecum. These data provide insights regarding the differences that distinguish the interactions of $C$. jejuni with chicken and human cells, and clearly show that chicken LMH cells respond differently to $C$. jejuni versus Salmonella typhimurium.

\section{METHODS}

Culture of bacterial strains. C. jejuni F38011 and 81-176 human clinical strains and C. jejuni D34a and A2a poultry strains were cultured under microaerobic conditions $\left(5 \% \mathrm{O}_{2}, 10 \% \mathrm{CO}_{2}, 85 \%\right.$ $\mathrm{N}_{2}$ ) on Mueller-Hinton agar plates supplemented with $5 \%(\mathrm{v} / \mathrm{v})$ citrated bovine blood (MH-blood agar). C. jejuni F38011 was recovered from an individual with diarrhoea containing blood and leukocytes in the stool sample. C. jejuni strain A2a was recovered from a Kansas poultry plant that processed organic broiler chickens. Organic poultry do not receive any coccidiostats, antibiotics, pesticides or insecticides (e.g. on their feed or in their environment), and must have access to the outside. C. jejuni strain D34a was 
recovered from an Iowa poultry processor plant from chickens reared using conventional methods. Cultures were passaged to a fresh $\mathrm{MH}-$ blood agar plate every 24-48 h. S. typhimurium 85-102840 and Escherichia coli XL1-Blue MRF' (Stratagene) were cultured on LuriaBertani (LB) agar plates.

Motility assay. Motility assays were performed using MH medium supplemented with $0.4 \%$ Select agar (Invitrogen). Briefly, $10 \mu \mathrm{l}$ of a bacterial suspension was added to the centre of a plate, and the plate was incubated at $37{ }^{\circ} \mathrm{C}$ under microaerobic conditions for $48 \mathrm{~h}$. The relative motility of each bacterial strain was determined by measuring the diameter of the migration zone.

Culture of LMH and INT 407 cells. LMH chicken hepatocellular carcinoma epithelial cells (ATCC CRL-2117), INT 407 human embryonic epithelial cells (ATCC CCL-6) and HepG2 cells (ATCC HB-8065) were obtained from the American Type Culture Collection. Stock cultures of LMH cells were grown in flasks coated with $0.1 \%$ gelatin in Waymouth's MB 752/1 medium supplemented with $10 \%$ fetal bovine serum (FBS). Stock cultures of INT 407 and HepG2 cells were grown in minimal essential medium (MEM) supplemented with $10 \%$ FBS (HyClone Laboratories). Cultures were maintained at $37{ }^{\circ} \mathrm{C}$ in a humidified, $5 \% \mathrm{CO}_{2}$ incubator.

C. jejuni-host cell binding and internalization assays. For the adherence assays, each well of a 24-well tissue-culture tray was seeded with LMH, INT 407 or HepG2 cells $\left(1.5-3.0 \times 10^{5}\right.$ cells per well $)$ and incubated for $18 \mathrm{~h}$ at $37{ }^{\circ} \mathrm{C}$ in a humidified, $5 \% \mathrm{CO}_{2}$ incubator. The cells were rinsed with the appropriate medium and inoculated with approximately $5 \times 10^{7}$ c.f.u. of the various $C$. jejuni strains. The cells were also inoculated with the same number of S. typhimurium and E. coli MRF', which were used as positive and negative controls, respectively. Bacteria-host cell contact was promoted by centrifugation at $600 \mathrm{~g}$ for $5 \mathrm{~min}$. Following a $30 \mathrm{~min}$ incubation at $37^{\circ} \mathrm{C}$ in a humidified, $5 \% \mathrm{CO}_{2}$ incubator, the epithelial cells were rinsed three times with PBS (20 mM sodium phosphate, $150 \mathrm{mM} \mathrm{NaCl}, \mathrm{pH} 6.9)$ to remove non-adherent bacteria. The epithelial cells were then lysed with a solution of $0.1 \%(\mathrm{v} / \mathrm{v})$ Triton X-100 in PBS. The suspensions were serially diluted and the number of viable, adherent bacteria determined by counting the resultant colonies on $\mathrm{MH}$-blood agar plates. For internalization, each 24 -well plate containing the $C$. jejuni-inoculated cells was incubated for $3 \mathrm{~h}$. The media were aspirated and replaced with culture media containing $1 \% \mathrm{FBS}$ and $250 \mu \mathrm{g}$ gentamicin $\mu \mathrm{l}^{-1}$. The plates were incubated for an additional $3 \mathrm{~h}$, and cell lysis and plating of serial dilutions were performed as outlined above. The values reported represent the mean counts \pm SD derived from triplicate wells.

RNA isolation from eukaryotic cell lines. For isolation of RNA from LMH or INT 407 cells, six-well plates were seeded with $1.2 \times 10^{6}$ and $6 \times 10^{5}$ cells, respectively. Bacterial suspensions were added at an m.o.i. of $1: 100$ in $6 \mathrm{ml} \mathrm{MEM} \mathrm{1 \%} \mathrm{FBS.} \mathrm{Samples} \mathrm{were} \mathrm{centrifuged} \mathrm{at}$ $600 \mathrm{~g}$ for $5 \mathrm{~min}$ and placed in a humidified $37^{\circ} \mathrm{C}$ incubator with $5 \%$ $\mathrm{CO}_{2}$ for $4 \mathrm{~h}$. Cells were washed once with ice-cold PBS and lysed with $1 \mathrm{ml}$ TRIzol reagent (Invitrogen). A cell scraper was used to remove adherent cells and the resulting lysate was vortexed thoroughly. For phase separation, $200 \mu \mathrm{l}$ chloroform: iso-amyl alcohol (24:1) was added, vortexed for $15 \mathrm{~s}$, incubated at room temperature for $3 \mathrm{~min}$, and spun at $14000 \mathrm{~g}$ for $15 \mathrm{~min}$ at $4{ }^{\circ} \mathrm{C}$. The upper aqueous layer was transferred to a fresh tube and precipitated with $0.5 \mathrm{ml}$ isopropyl alcohol. To collect the RNA precipitate, samples were centrifuged at $14000 \mathrm{~g}$ for $10 \mathrm{~min}$ at $4{ }^{\circ} \mathrm{C}$. The supernatant was discarded and the pellet was washed twice with $75 \%$ ethanol and resuspended in $100 \mu \mathrm{l}$ nuclease-free water. Samples were loaded on a $2 \%$ agarose gel to verify extraction efficacy prior to RNA cleanup using the RNeasy kit (Invitrogen) according to the manufacturer's instructions. RNA samples were generated on three separate days and used for reversetranscription reactions.
Real-time RT-PCR of IL-8. Reverse transcription of RNA extracts was performed using the ThermoScript RT-PCR System (Invitrogen) according to the manufacturer's protocol. Briefly, $1 \mu \mathrm{g}$ total mRNA was combined with $50 \mu \mathrm{M}$ Oligo $(\mathrm{dT})_{20}$ random primer in a $20 \mu \mathrm{l}$ total volume with ThermoScript reverse transcriptase. Thermal reaction conditions were as follows: preheating of the RNA sample for $5 \mathrm{~min}$ at $65{ }^{\circ} \mathrm{C}$, addition of the reaction mixture on ice, heating for $60 \mathrm{~min}$ at $50{ }^{\circ} \mathrm{C}, 85^{\circ} \mathrm{C}$ for $5 \mathrm{~min}$ for enzyme denaturation, and rapid cooling to $4{ }^{\circ} \mathrm{C}$. Real-time RT-PCR analysis was performed with three cDNA sample sets.

Transcript quantification was performed on the ABI Prism 7000 Sequence Detection System (Applied Biosystems) with primers and PCR conditions described elsewhere (Borrmann et al., 2007). Realtime quantitative PCR was carried out with $7.5 \mathrm{ng}$ CDNA template using the following primers: chCXCLi2 (CAF) Fwd 5'-ATG AAC GGC AAG CTT GGA GCT-3', Rev 5'-GCC ATA AGT GCC TTT ACG ATC AG-3'; chCXCLi1 (K60) Fwd 5'-TGG CTC TTC TCC TGA TCT CAA TG-3', Rev 5'-GCA CTG GCA TCG GAG TTC A-3'; and chicken beta actin Fwd $5^{\prime}$-CAT CAC CAT TGG CAA TGA GAG G-3', Rev 5'-GAT TCA TCG TAC TCC TGC TTG C-3'; huCXCL8 Fwd 5' ATG ACT TCC AAG CTG GCC GTG GCT-3', Rev 5'-TCT CAG CCC TCT TCA AAA ACT TCT C-3'; human GAPDH Fwd 5'-TGG GTG TGA ACC ATG AGA AG-3', Rev 5'-GCT AAG CAG TTG GTG GTG C-3'. For the chIL- 8 and beta actin samples, $450 \mathrm{nM}$ of each primer was used; and for huCXCL8 and GAPDH samples, $300 \mathrm{nM}$ of each primer was used. Duplicate $50 \mu \mathrm{l}$ reactions were performed with the Power SYBR Green PCR Master Mix (Applied Biosystems) for each sample with each primer set. RNA samples without a reverse transcriptase step were included for every sample and primer combination to ensure the absence of genomic DNA. Thermal cycling conditions for RT-PCR were as follows: $2 \mathrm{~min}$ at $50{ }^{\circ} \mathrm{C}$, 15 min at $95{ }^{\circ} \mathrm{C}$ followed by 40 repeats of $15 \mathrm{~s}$ at $94{ }^{\circ} \mathrm{C}, 30 \mathrm{~s}$ at $59{ }^{\circ} \mathrm{C}$, and $30 \mathrm{~s}$ at $72{ }^{\circ} \mathrm{C}$. PCR efficiencies were determined from standard curves of cDNA dilutions and their respective cycle thresholds $\left(\mathrm{C}_{t} \mathrm{~s}\right)$. A reference gene was used to correct for variance in mRNA template concentration and efficiency of the reverse-transcription reaction. The chicken beta actin gene was used as the reference gene for chIL- 8 and the human GAPDH gene was used as the reference gene for $h u$ CXCL8. These data were analysed using the comparative $\mathrm{C}_{\mathrm{t}}$ method, and the results are presented as the fold increase of gene expression of the bacteria-inoculated samples relative to the uninoculated sample, as described by Applied Biosystems.

\section{Generation of $\boldsymbol{C}$. jejuni-inoculated LMH and INT 407 cell culture} supernatant fluids. Overnight cultures of bacteria were washed twice by centrifugation for $10 \mathrm{~min}$ at $8000 \mathrm{~g}$ and resuspended in the appropriate tissue-culture medium supplemented with $1 \%$ FBS. The epithelial cells were co-cultured with $0.5 \mathrm{ml}$ (per well) of a bacterial suspension containing a $1: 10$ dilution of $5 \times 10^{7}$ c.f.u. of the appropriate C. jejuni strain. The plate was centrifuged at $600 \mathrm{~g}$ for 5 min before being transferred to a $37{ }^{\circ} \mathrm{C}$ incubator. Supernatant fluids were collected $24 \mathrm{~h}$ after inoculation, and stored at $-20{ }^{\circ} \mathrm{C}$. Supernatant fluids were also collected from uninoculated cells as a negative control and cells inoculated with a $1: 1000$ dilution of $5 \times 10^{7}$ c.f.u. S. typhimurium as a positive control. In certain instances, a solution of phorbol 12-myristate 13-acetate and ionomycin (PMA/I, $10 \mathrm{pg} \mathrm{ml}^{-1}$ of each) was included as a separate, stimulation (positive) control.

IL-8 ELISAs. ELISA was performed to detect the presence of IL-8 secreted by LMH or INT 407 cells. Polystyrene plates (96-well, Costar) were coated with $50 \mu \mathrm{l}$ per well of $0.4 \mu \mathrm{g} \mathrm{ml}^{-1}$ mouse antihuCXCL8 (Pierce Endogen) and stored at $4{ }^{\circ} \mathrm{C}$ overnight under humid conditions. Plates were blocked with $150 \mu \mathrm{l} 1 \%$ (w/v) BSA (fraction V, Sigma) in PBS for $30 \mathrm{~min}$ at room temperature. After blocking, plates were washed three times with PBS containing Tween 
$20(0.005 \% \mathrm{v} / \mathrm{v})$ (PBS-Tween). Supernatant fluid samples and chicken sera (diluted $1: 10$ in PBS) were added at $100 \mu \mathrm{l}$ per well and incubated for $2 \mathrm{~h}$ at room temperature. Wells were washed three times with PBS-Tween, after which $100 \mu \mathrm{l}$ of $0.1 \mu \mathrm{g} \mathrm{ml} \mathrm{m}^{-1}$ biotinlabelled mouse anti-huCXCL8 (Pierce Endogen) in PBS with 1\% BSA was added. Samples were incubated for $1 \mathrm{~h}$ at room temperature. The plate was washed three times with PBS-Tween and $100 \mu \mathrm{l}$ of $0.25 \mu \mathrm{g}$ $\mathrm{ml}^{-1}$ horseradish peroxidase conjugated streptavidin (SA-HP) in PBS with $1 \%$ BSA was added. After a $30 \mathrm{~min}$ incubation period, the plate was washed three times with PBS-Tween. Fifty microlitres of 3,3, $5^{\prime}, 5^{\prime}$ tetramethylbenzidine (TMB; Pierce) per well was then added and allowed to incubate for $15 \mathrm{~min}$. Colorimetric development was stopped by adding $50 \mu \mathrm{l} 0.18 \mathrm{M} \mathrm{H}_{2} \mathrm{SO}_{4}$. $A_{450}$ was read with a Titertek Multiscan MCC/340. All experimental samples were assayed in triplicate. Control samples of recombinant huCXCL8 (Pierce Endogen) ranging from 20 to $5000 \mathrm{pg} \mathrm{ml}^{-1}$ were included as an internal control for reproducibility and dose-dependent response. The mean and SDs of the absorbance readings were calculated from triplicate measurements. Statistical analysis to determine significance was performed using Student's $t$ test.

To demonstrate specificity of the chIL-8 ELISA, one microtitre plate (plate 1) was coated with $50 \mu \mathrm{l}$ mouse anti-huCXCL8 antibody (Pierce Endogen) in serial twofold dilutions ranging from 7 to $1000 \mathrm{pg} \mathrm{ml}^{-1}$ and incubated overnight at $4{ }^{\circ} \mathrm{C}$. A second ELISA plate (plate 2) was coated with $50 \mu \mathrm{l}$ per well $0.4 \mu \mathrm{g} \mathrm{ml}^{-1}$ mouse antihuCXCL8 and stored at $4{ }^{\circ} \mathrm{C}$ overnight, as above. A $100 \mu \mathrm{l}$ volume of S. typhimurium-inoculated LMH supernatant fluids was added to plate 1 for $45 \mathrm{~min}$ at room temperature. Supernatant fluids were then transferred to plate 2 . Both plate 1 and plate 2 were blocked and analysed for bound chIL-8 as described above. Specificity was demonstrated by the dose-dependent capture of chIL-8 on plate 1 , and the inverse trend of chIL- 8 capture on plate 2 .

Heterophil cell migration assays. One-day-old broiler chickens were obtained from a commercial source (Phinney Hatchery), raised in wired cages, and given free access to feed and water. At 3-4 weeks of age, $3 \mathrm{ml}$ blood was drawn from the brachial wing vein using a sterile plastic syringe containing $0.3 \mathrm{ml} 10 \%$ disodium EDTA. Heterophils were isolated from blood using a published technique (Andreasen \& Latimer, 1989) with some modifications. Briefly, for each $1.5 \mathrm{ml}$ of EDTA-anticoagulated blood, an equal volume of $1 \%$ $(w / v)$ methylcellulose was added, mixed, and centrifuged at $25 \mathrm{~g}$ for $10 \mathrm{~min}$. The plasma and extended buffy-coat layers were removed, diluted in 0.5-1 ml serum-free Dulbecco's Modified Eagle's Medium (MEM; Invitrogen) and layered on to a discontinuous FicollHypaque gradient ( $3 \mathrm{ml}$ at specific gravity $1.077 ; 3 \mathrm{ml}$ at 1.119 ) (Sigma). Following centrifugation at $250 \mathrm{~g}$ for $35 \mathrm{~min}$, the plasma1.077 interface was discarded. The remaining gradient (partial 1.077 and 1.119) containing heterophils was collected and washed three times in serum-free MEM. Viable cells were counted using a haemocytometer and resuspended in serum-free MEM to achieve concentration of $1-2 \times 10^{4}$ cells per $100 \mu \mathrm{l}$.

The presence of chemoattractants in the supernatant fluids collected from C. jejuni-inoculated LMH cells was assessed by chicken heterophil cell migration with a QCM Chemotaxis 96-well Cell Migration Assay kit ECM515 (Chemicon). Briefly, freshly isolated chicken heterophils $\left(2 \times 10^{4}\right)$ in $100 \mu \mathrm{l}$ serum-free medium were loaded into the upper migration chamber of the transwell plate and $150 \mu \mathrm{l}$ of supernatant from C. jejuni-inoculated LMH cells was added to the lower chamber (feeder tray). Supernatant fluids were collected from uninoculated and S. typhimurium-inoculated cells as negative and positive controls, respectively. Cells were incubated in a humidified, $5 \% \mathrm{CO}_{2}$ incubator for $45 \mathrm{~min}$ at $37^{\circ} \mathrm{C}$. After incubation, the upper chamber was incubated with $150 \mu \mathrm{l}$ prewarmed cell detachment solution for $30 \mathrm{~min}$ at $37^{\circ} \mathrm{C}$ to dislodge migrated cells from the underside of the insert. From the feeder tray, $75 \mu \mathrm{l}$ of the medium was transferred to a 96-well plate and $75 \mu 1$ of the cell detachment solution was transferred to the same plate. To this $150 \mu \mathrm{l}$ mixture, $50 \mu \mathrm{l}$ lysis buffer/dye solution containing CyQuant GR dye was added. The fluorescence was measured using a Wallac 1420 fluorescence plate reader set at excitation/emission wavelengths of $485 / 535 \mathrm{~nm}$, and the total number of migrated heterophils in each sample was calculated using a standard curve obtained for known concentrations of heterophils. All samples were tested in triplicate, and the statistical significance between samples was determined using Student's $t$ test $(P<0.05)$.

Inoculation of chickens. Thirty-six nine-day-old broiler chickens were divided into six groups and placed into isolation chambers (Horsfall-Bauer isolators) on wire mesh. Water and a commercial chick starter feed were provided ad libitum. The chickens were inoculated by oral gavage with $0.5 \mathrm{ml}$ of a suspension of $C$. jejuni F38011 strain, which was cultured in Bolton's broth at $42{ }^{\circ} \mathrm{C}$ for $16 \mathrm{~h}$ under microaerobic conditions. One group of six chickens was kept as the uninoculated control group. The remaining five groups of chickens were inoculated with: (1) $10^{2}$ c.f.u. C. jejuni F38011; (2) $10^{3}$ c.f.u. C. jejuni F38011; (3) $10^{4}$ c.f.u. C. jejuni F38011; (4) $10^{5}$ c.f.u. C. jejuni F38011; and (5) $10^{6}$ c.f.u. C. jejuni F38011. The bacterial suspensions were serially diluted and plated onto cefoperazonevancomycin-amphotericin B (CVA) agar to confirm the c.f.u. of each dose. Six days later the chickens were euthanized, and the number of C. jejuni per caecal sample was determined by direct plating. More specifically, the caecum with its contents was collected, placed into a whirl-pack, sliced open, weighed, diluted $1: 10(\mathrm{w} / \mathrm{v})$ in Bolton's broth medium, and thoroughly stomached. The serial 10-fold dilutions were made in $3 \mathrm{ml}$ quantities of Bolton's broth medium. The diluted samples were spread onto the surface of CVA agar plates and incubated in a microaerobic environment at $37^{\circ} \mathrm{C}$. The c.f.u. were determined for each sample after $48 \mathrm{~h}$ of incubation. Blood was also collected from each chicken.

A second in vivo colonization experiment was performed with 12 broiler chickens. Six chickens were inoculated with $10^{7}$ c.f.u. C. jejuni F38011 strain and six uninoculated chickens were used as a control. Three of the C. jejuni-inoculated chickens and three uninoculated chickens were euthanized at 8 days post-inoculation (p.i.), and the remaining six chickens at 15 days p.i. Caecal and blood samples were collected as described above. All of the chicken studies and procedures were performed in compliance with protocols approved by the Institutional Animal Care and Use Committee (IACUC protocol \#3248) at Washington State University.

Immunoblot analysis and ELISA of chicken serum antibodies. Blood samples were collected from chickens sacrificed 6, 8 and 15 days p.i., as described above in the inoculation protocol. Sera were separated by centrifugation and stored at $-20{ }^{\circ} \mathrm{C}$. For immunoblot analysis, C. jejuni F38011 whole-cell lysates were normalized to 0.1 $\mathrm{OD}_{540}$ units in electrophoresis sample buffer and incubated in a boiling water bath for $5 \mathrm{~min}$. The proteins were separated in a $12 \%$ acrylamide gel matrix using Laemmli's discontinuous buffer system and transferred to PVDF membranes (Immobilon P, Millipore). The membranes were rinsed in PBS and incubated for $16 \mathrm{~h}$ at room temperature with a 1:50 dilution of chicken sera in PBS ( $\mathrm{pH} 7.4)$ containing $0.01 \%$ Tween-20 and $9 \%(\mathrm{w} / \mathrm{v})$ dried milk. Bound immunoglobulins were detected with either anti-chicken IgM (ICL) or anti-chicken IgG horseradish peroxidase conjugates (Sigma). 4Chloro-1-naphthol (Sigma) was used as the chromogenic substrate. The sera were also tested for anti-C. jejuni antibodies by ELISA. For these assays, 96-well microtitre plates (Costar 3797, Corning) were coated with $1 \mu \mathrm{g}$ C. jejuni F38011 whole-cell lysate overnight at $4{ }^{\circ} \mathrm{C}$. After blocking with PBS $/ 0.5 \%$ BSA (w/v) for $2 \mathrm{~h}$ at room temperature, the chicken sera were added in twofold serial dilutions 
(ranging from $1: 50$ to $1: 6400$ ) and incubated for $2 \mathrm{~h}$ at room temperature. The wells were rinsed three times with PBS-Tween and incubated for $30 \mathrm{~min}$ with a $1: 1000$ dilution of anti-chicken IgGhorseradish peroxidase antibody (Sigma). The plates were developed as described above.

\begin{abstract}
Histopathology and transmission electron microscopy (TEM). Intestinal and caecal tissues were collected at the time of necropsy and placed in $10 \%$ buffered formalin. Each sample was coded to conceal whether it was collected from a C. jejuni-inoculated or uninoculated chicken, and given to the core facility for processing. The samples were prepared with an automatic processor (Tissue-Tek VP, Sakura) and embedded in paraffin, and $4 \mu \mathrm{m}$ thick sections were mounted onto glass slides. The sections were de-paraffinized with xylene and stained with haematoxylin and eosin. All the samples were examined by a board-certified pathologist. The sample identification code was shared with the pathologist after the final summary report was provided to the investigators. In total, intestinal and caecal tissues were examined from two control chickens (i.e. uninoculated) and two C. jejuni-inoculated chickens euthanized at 8 days p.i., and from one control chicken and one C. jejuni-inoculated chicken euthanized at 15 days p.i.
\end{abstract}

For TEM, chicken caecum samples were removed from $10 \%$ formalin, cut into $1 \times 2 \mathrm{~mm}$ pieces and placed in $2 \%$ paraformaldehyde/ $2 \%$ glutaraldehyde in $0.1 \mathrm{M}$ cacodylate buffer overnight at $4{ }^{\circ} \mathrm{C}$. Samples were rinsed in buffer and post-fixed in $2 \% \mathrm{OsO}_{4} 0.1 \mathrm{M}$ cacodylate buffer overnight at $4{ }^{\circ} \mathrm{C}$ and dehydrated in an ethanol series, followed by two changes of propylene oxide. Samples were infiltrated with $1: 1$ Spurr's epoxy resin for $2 \mathrm{~h}$, followed by two changes with $100 \%$ Spurr's epoxy resin, and polymerized overnight in $100 \%$ Spurr's $2 \times$ at $70{ }^{\circ} \mathrm{C}$. Thin sections were collected on nickel grids using a Reichert Ultra Cut $\mathrm{R}$ ultramicrotome (Leica Microsystems), stained with $4 \%$ uranyl acetate $/ 1 \%$ potassium and Reynold's Lead, and viewed with a JEOL JEM 1200 EX transmission electron microscope. Images were obtained with a MegaView III camera using analySIS 3.2 software [Olympus Soft Imaging Systems Corp. (SIS)].

\section{RESULTS}

\section{C. jejuni is less invasive of LMH cells compared with human INT 407 cells}

Binding and internalization assays were performed with four C. jejuni strains and chicken LMH hepatocellular carcinoma epithelial cells. Two C. jejuni strains (F38011 and 81-176) were recovered from individuals exhibiting clinical signs of campylobacteriosis, and two C. jejuni strains (A2a and D34a) were recovered from poultry processing plants. All four C. jejuni strains were motile (not shown). Earlier work has indicated that S. typhimurium efficiently invades primary chick cells, while a laboratory strain of E. coli poorly invades these cells (Kaiser et al., 2000). In this study, S. typhimurium 85-102840 was used as a positive control for host cell invasion and E. coli XL1Blue $\mathrm{MRF}^{\prime}$ as a negative control. Assays were performed in parallel with human INT 407 epithelial cells.

The results of the bacterial binding assays are shown in Table 1. Similar numbers of S. typhimurium and E. coli bound to the LMH and INT 407 cells. In addition, all four C. jejuni strains bound LMH cells and INT 407 cells in comparable numbers $(P>0.05)$. The variations in adherence among the four $C$. jejuni strains to the LMH cells did not correlate with the isolation source of the strain (i.e. human versus poultry). These data indicated that $C$. jejuni binding to the host epithelial cells is independent of host cell species (i.e. chicken versus human cells).

The results of the bacterial invasion assays are shown in Table 1. S. typhimurium invaded LMH cells at $4.5 \pm$ $1.9 \times 10^{5}$ c.f.u. per well and invaded INT 407 cells at $6.2 \pm 0.9 \times 10^{5}$ c.f.u. per well. E. coli invaded LMH cells at $2.9 \pm 0.5 \times 10^{3}$ c.f.u. per well and invaded INT 407 cells at $2.8 \pm 0.6 \times 10^{3}$ c.f.u. per well. There was no significant difference in the numbers of either S. typhimurium or $E$. coli that invaded the LMH cells when compared with INT 407 cells. In contrast to the controls, the number of $C$. jejuni that invaded LMH cells was significantly lower $(P<0.05)$ than the number of $C$. jejuni that invaded the INT 407 cells. Because the number of $C$. jejuni that invade host cells is dependent on the number bound, we calculated I/A values [(number of intracellular bacteria/ number of cell-associated bacteria) $\times 100$ ] to normalize the number of internalized bacteria relative to the number of

Table 1. Binding and internalization of $C$. jejuni human clinical and poultry strains to LMH chicken hepatocellular carcinoma epithelial cells and INT 407 human embryonic epithelial cells

\begin{tabular}{|c|c|c|c|c|c|c|}
\hline \multirow[t]{2}{*}{ Isolate } & \multicolumn{3}{|c|}{ LMH cells } & \multicolumn{3}{|c|}{ INT 407 cells } \\
\hline & $\begin{array}{l}\text { Number of cell-asso- } \\
\text { ciated bacteria }\end{array}$ & $\begin{array}{l}\text { Number of intern- } \\
\text { alized bacteria } \dagger\end{array}$ & $\begin{array}{c}(\mathrm{I} / \mathrm{A}) \times 100 \\
(\%) \ddagger\end{array}$ & $\begin{array}{l}\text { Number of cell-asso- } \\
\text { ciated bacteria }^{*}\end{array}$ & $\begin{array}{l}\text { Number of intern- } \\
\text { alized bacteria } \dagger\end{array}$ & $\begin{array}{c}(\mathrm{I} / \mathrm{A}) \times 100 \\
(\%) \neq\end{array}$ \\
\hline S. typhimurium & $(1.2 \pm 0.1) \times 10^{7}$ & $(4.5 \pm 1.9) \times 10^{5}$ & 3.8 & $(1.3 \pm 0.2) \times 10^{7}$ & $(6.2 \pm 0.9) \times 10^{5}$ & 4.8 \\
\hline C. jejuni F38011 & $(9.6 \pm 1.0) \times 10^{6}$ & $(1.3 \pm 0.4) \times 10^{4}$ & 0.1 & $(9.8 \pm 1.5) \times 10^{6}$ & $(3.3 \pm 0.6) \times 10^{5}$ & 3.4 \\
\hline C. jejuni 81-176 & $(6.6 \pm 2.1) \times 10^{6}$ & $(1.2 \pm 0.7) \times 10^{4}$ & 0.2 & $(7.8 \pm 1.0) \times 10^{6}$ & $(6.2 \pm 0.4) \times 10^{5}$ & 8.0 \\
\hline E. coli MRF & $(1.4 \pm 0.2) \times 10^{7}$ & $(2.9 \pm 0.5) \times 10^{3}$ & 0.02 & $(1.3 \pm 0.1) \times 10^{7}$ & $(2.8 \pm 0.6) \times 10^{3}$ & 0.02 \\
\hline
\end{tabular}

${ }^{*}$ Number of cell-associated bacteria per well of a 24 -well plate.

$\dagger$ Number of internalized bacteria per well of a 24 -well plate, as determined by the gentamicin-protection assay.

$\$(\mathrm{I} / \mathrm{A}) \times 100$ indicates the percentage of cell-associated bacteria that were internalized. 
adherent bacteria for the different strains used in this study. Comparisons of S. typhimurium, E. coli and C. jejuni I/A values for the LMH and INT 407 cells revealed that while C. jejuni does invade LMH chicken cells, it is significantly less invasive for LMH cells than INT 407 cells. Collectively, we concluded that each C. jejuni strain used in this study: (1) exhibited similar binding potential to LMH and INT 407 cells; (2) demonstrated a low invasive potential for LMH cells and a high invasive potential for INT 407 cells; and (3) variations in binding and invasion of the $C$. jejuni strains did not correlate with the source (i.e. human versus poultry) of the isolate. We concluded that each of the four $C$. jejuni strains is virulent based on the fact that the C. jejuni strains invaded the human INT 407 cells in numbers that were similar with one another and that of the S. typhimurium strain, and two of the four C. jejuni strains were recovered from individuals with campylobacteriosis.

\section{C. jejuni stimulates greater chCXCLi1 expression than chCXCLi2 in LMH cells}

Real-time RT-PCR was used to measure chCXCLil, chCXCLi2 and huCXCL8 transcripts in epithelial cells inoculated with $C$. jejuni or S. typhimurium, PMA/I treated cells (i.e. positive stimulation control), and media only-treated cells (i.e. mock-inoculated, negative stimulation control) (Table 2). Relative to the uninoculated LMH cells, S. typhimurium induced a $13.04 \pm 1.44$-fold increase and a $21.18 \pm 5.52$-fold increase in chCXCLi1 and chCXCLi2 expression, respectively. Each of the four $C$.

Table 2. Analysis of chCXCLi2 (CAF/IL-8) and chCXCLi1 (K60) expression in LMH cells, and huCXCL8 expression in INT 407 cells

The relative fold changes in transcript levels were determined from $C$. jejuni- and S. typhimurium-inoculated cells using the comparative $\mathrm{C}_{\mathrm{t}}$ method. All samples were normalized as described in Methods.

\begin{tabular}{|lcc|}
\hline \multirow{2}{*}{ Bacterial isolate } & \multicolumn{2}{c|}{ Relative fold change of transcript } \\
\cline { 2 - 3 } & chCXCLi1 $($ K60) & chCXCLi2 $($ CAF $)$ \\
\hline Chicken LMH cells & & \\
$81-176$ & $16.84 \pm 3.25$ & $2.87 \pm 0.77$ \\
F38011 & $3.84 \pm 0.53$ & $2.52 \pm 1.13$ \\
D34a & $6.05 \pm 2.06$ & $1.25 \pm 0.32$ \\
A2a & $28.45 \pm 5.87$ & $3.56 \pm 2.30$ \\
85-102840 & $13.04 \pm 1.44$ & $21.18 \pm 5.52$ \\
PMA/I* & $596.16 \pm 194.77$ & $92.41 \pm 10.86$ \\
\hline & huCXCL8 \\
\hline Human INT 407 cells & \\
81-176 & $1283.94 \pm 201.29$ \\
85-102840 & $39.67 \pm 0.41$ & \\
PMA/I* & $11.80 \pm 0.12$ \\
\hline
\end{tabular}

${ }^{\star} \mathrm{PMA} / \mathrm{I}$ was included as a separate, stimulation-positive control. jejuni strains induced the expression of both chCXCLi1 and chCXCLi2. The greatest level of chCXCLi1 and chCXCLi2 expression from the C. jejuni-inoculated cells was noted for strain A2a. The expression of chCXCLi2 in the C. jejuni A2a-inoculated LMH cells was significantly less than that observed with S. typhimurium. Also, in contrast with S. typhimurium, the expression of chCXCLi1 relative to chCXCLi2 was greater in the $C$. jejuniinoculated LMH cells. Noteworthy is the fact that the level of $h u C X C L 8$ expression in INT 407 cells inoculated with C. jejuni 81-176 was greater than huCXCL8 expression induced by $S$. typhimurium. Based on these assays, we concluded that the expression of huCXCL8 orthologues in chicken LMH cells in response to $C$. jejuni is different from that of S. typhimurium.

\section{C. jejuni does not induce chicken IL-8 secretion from LMH cells, as judged by ELISA}

Due to the unavailability of antibodies against chCXCLi1 and chCXCLi2, we used capture and detection antibodies generated against human IL8 (huCXCL8) in a sandwich ELISA, with the caveat that it is not known whether these antibodies would detect either chCXCLi1 or chCXCLi2. Thus, we refer to the protein detected by the ELISA as 'chIL-8' rather than chCXCLi1 or chCXCLi2. ELISAs were performed with supernatant fluids collected from epithelial cells inoculated with C. jejuni 81-176 or S. typhimurium, and media only-treated cells (i.e. uninoculated cells) (Fig. 1). Epithelial cells were inoculated and $24 \mathrm{~h}$ after inoculation supernatant fluids were collected. LMH cells incubated with $S$. typhimurium released significant amounts of chIL-8. In contrast, a significant difference was not observed in chIL-8 in the supernatants from LMH cells inoculated with C. jejuni strain 81-176 when compared with cells treated with media only. Recombinant huCXCL8 protein standards were run in parallel to assess dose-dependency and specificity of the sandwich ELISA. Additional assays revealed that none of the four $C$. jejuni strains induced the release of detectable amounts of chIL-8 from LMH cells, but each stimulated huCXCL8 protein secretion from the INT 407 cells (results not shown). To further demonstrate that the huCXCL8 antibody used in the sandwich ELISA reacts with chIL-8, the supernatant fluids collected from S. typhimuriuminoculated LMH cells were incubated in wells of an ELISA plate coated with serial dilutions of the capture antibody. Specificity of the reaction was demonstrated by the observation that as the concentration of capture antibody increased, a decrease was observed in the amount of chIL-8 detected in the supernatant fluid (results not shown). It is not known whether the capture antibody reacts with either chCXCLi1 and/or chCXCLi2. Inoculation of the INT 407 cells with both $C$. jejuni and $S$. typhimurium induced huCXCL8 secretion (Fig. 1). The IL-8 secretion responses obtained with HepG2 cells were similar to those of the INT 407 cells (results not shown). 


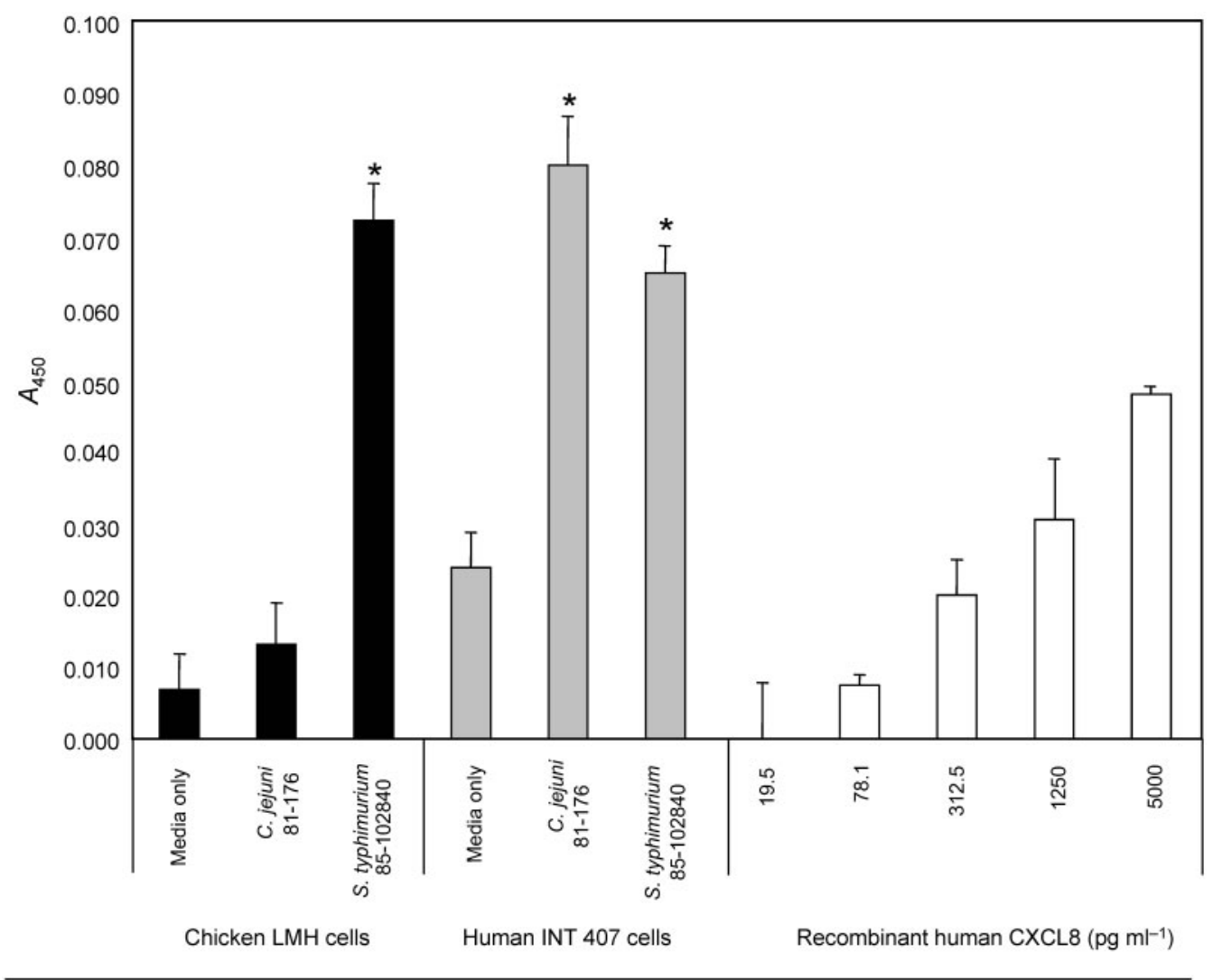

* Indicates that the absorbance value was significantly different from the media-only control $(P<0.05)$.

Fig. 1. C. jejuni 81-176 does not elicit chlL-8 secretion from LMH cells, as judged by ELISA. C. jejuni was incubated with LMH and INT 407 cell monolayers for 24 h, and supernatant fluids were assayed for IL-8 as outlined in Methods. S. typhimurium $85-$ 102840 -inoculated cells were used as a positive control and uninoculated cells (media only) were used as a negative control for IL-8 induction.

\section{Supernatants from $C$. jejuni-inoculated LMH cells stimulate minimal chemotactic activity}

Supernatant fluids were collected from LMH cells inoculated with $C$. jejuni at $24 \mathrm{~h}$ p.i. and used for the heterophil migration assays (Fig. 2). Supernatants of LMH cells inoculated with $S$. typhimurium and uninoculated cells were used as positive and negative controls, respectively. The supernatant fluids from the LMH cells inoculated with C. jejuni and S. typhimurium induced heterophil migration. However, the S. typhimurium supernatants induced the migration of significantly more heterophils than the $C$. jejuni supernatants $(P<0.05)$.

\section{C. jejuni-colonized chickens produce anti- Campylobacter antibodies but not detectable chicken IL-8}

A preliminary experiment revealed that a dose of $10^{6} \mathrm{C}$. jejuni $\mathrm{F} 38011$ is sufficient to obtain $100 \%$ colonization of chickens (not shown). A second experiment was performed to assess antibody and chIL- 8 production in C. jejuni-inoculated chickens. All chickens inoculated with C. jejuni were colonized at 8 and 15 days p.i., whereas all of the uninoculated chickens were Campylobacter-free (Fig. 3a). IgG and IgM antibodies reactive against $C$. jejuni were detected in the serum of the C. jejuni-colonized chickens at 15 days p.i., but not at 8 days p.i., as judged by immunoblot analysis (not shown). ELISAs were performed to further assess the antibody responses of the chickens. The amount of antiCampylobacter antibodies in the sera from the C. jejunicolonized chickens at 15 days p.i. was significantly greater than that in the sera from uninoculated chickens at 15 days (Fig. 3b). In contrast, the amount of antiCampylobacter antibodies in the sera collected from the C. jejuni-colonized chickens at 8 days p.i. was not significantly different from the amount for the sera of the uninoculated chickens at 15 days. chIL-8 was not detected in any of the serum samples, as judged by ELISA. 


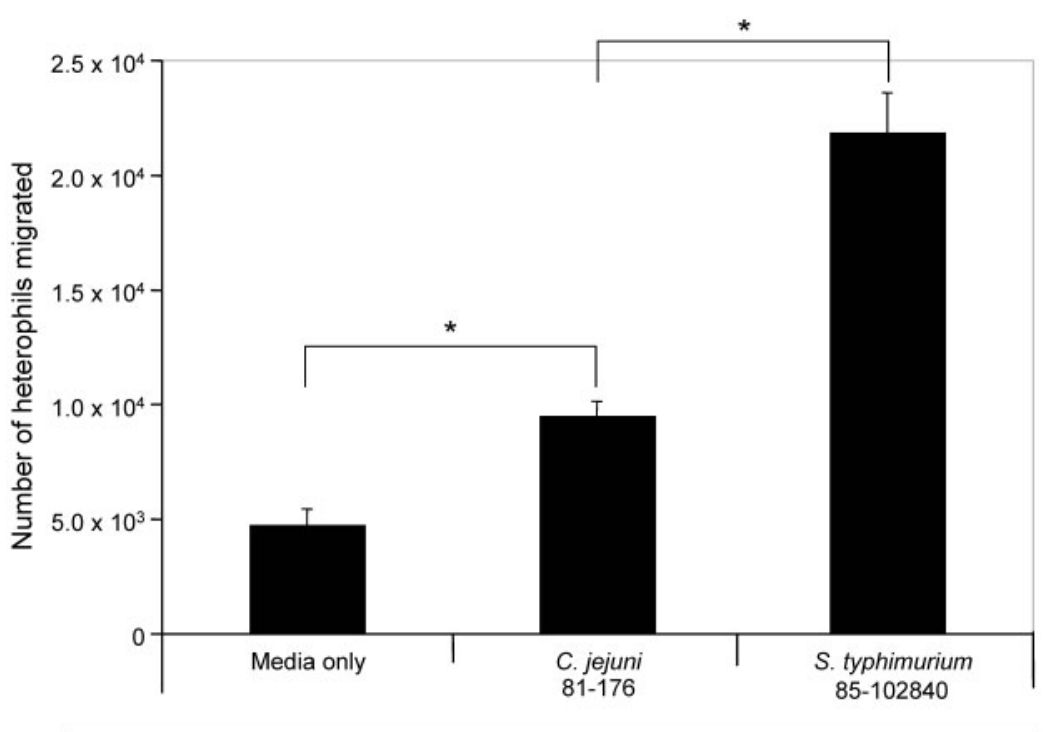

* Indicates significant difference $(P<0.05)$
Fig. 2. Chicken heterophil cell migration assays were performed with supernatant fluids of C. jejuni 81-176-inoculated LMH cells to assess the presence of cytokines. Supernatant fluids from S. typhimurium 85-102840-inoculated LMH cells and uninoculated cells (media only) were used as positive and negative controls for heterophil cell migration, respectively.

\section{C. jejuni F38011 does not result in overt pathology in the caeca of chickens}

Intestinal and caecal tissues were examined from the $C$. jejuni-inoculated and uninoculated chickens euthanized at 8 and 15 days p.i. None of the tissues collected from the $C$. jejuni-inoculated chickens revealed signs of histopathology. Shown is a representative caecal tissue sample from a chicken colonized with C. jejuni F38011 at 15 days p.i. (Fig. 4). Rod-shaped bacteria were observed in the deep colonic crypts of both the C. jejuni-inoculated and uninoculated chickens, but Campylobacter organisms were only recovered from the C. jejuni-inoculated chickens. None of the chickens exhibited epithelial necrosis, epithelial hyperplasia, or infiltration of heterophils, lymphocytes or plasma cells into the stroma of the lamina propria. Examination of the caecal tissues by TEM revealed numerous $C$. jejuni present in the glandular crypts of the inoculated chickens, but no bacteria were observed in the surrounding tissue (Fig. 5). C. jejuni were also observed in direct contact with the host tissue (Fig. 5c, d).

\section{DISCUSSION}

This study was conducted to further characterize the commensal-type interactions of C. jejuni in chickens and to compare these results with the pathogen-type interactions of $C$. jejuni with human cells. We hypothesized that the $C$. jejuni strains used in this study would behave similarly in a particular cell line, but their behaviour might differ depending on the host cell species (i.e. chicken or human). We selected four C. jejuni strains and assessed their interactions with chicken LMH hepatocellular carcinoma epithelial cells. The C. jejuni F38011 and 81-176 strains were recovered from humans with campylobacteriosis and the C. jejuni A2a and D34a strains were recovered from poultry-processing plants. Many of the experiments were conducted with human INT 407 embryonic epithelial cells to quantitatively compare the virulence behaviour of $C$. jejuni strains with chicken cells versus human cells. This study differs in two fundamental aspects from earlier studies designed to characterize the interaction of C. jejuni with chicken cells. First, we used a continuous chicken epithelial cell line (LMH) as an in vitro model to examine the interactions of C. jejuni with chicken cells. Second, we concomitantly assessed chCXCLi2 and chCXCLi1 expression, chIL-8 secretion, and production of functional chemoattractants from the chicken LMH epithelial cells inoculated with C. jejuni. Noteworthy is that the LMH cell line is the only chicken epithelial cell line currently available to researchers.

The C. jejuni strains used in this study were first tested for their ability to bind to LMH chicken hepatocellular carcinoma and INT 407 human embryonic epithelial cells. While the number of bacteria bound to the epithelial cells varied between the four $C$. jejuni strains, the differences in bacteria-host cell binding did not correlate with the source of a bacterial strain. The binding assays also revealed that the host cell species had little quantitative effect on the binding of $C$. jejuni to host cells. The results from the binding assays did not identify distinguishing characteristics to explain why C. jejuni is a commensal microbe in chickens and a pathogen in humans.

Striking differences were observed in the invasiveness of $C$. jejuni with LMH cells when compared with the INT 407 cells. The number of C. jejuni internalized by the LMH cells was significantly lower than the number of $C$. jejuni internalized by the INT 407 cells $\left(7 \times 10^{3}-1.6 \times 10^{4}\right.$ versus $2.8 \times 10^{5}-6.2 \times 10^{5}$, respectively). For all four $C$. jejuni strains, the percentage of adherent bacteria internalized by the chicken cells was reduced more than 20 times 


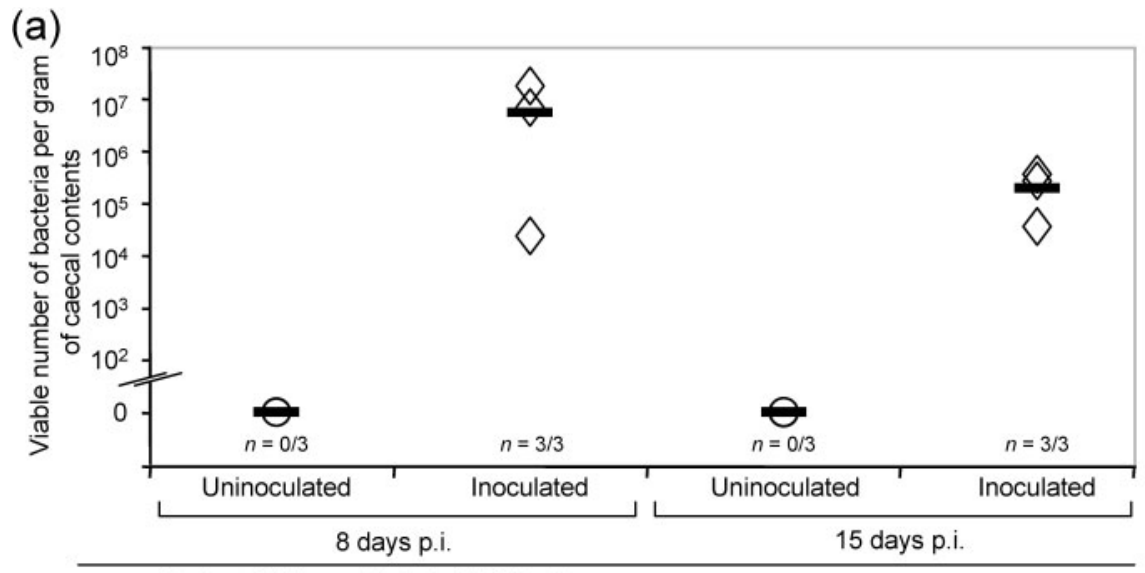

$n=$ Number of chickens colonized with $C$. jejuni

= Mean number of c.f.u. recovered from three chickens

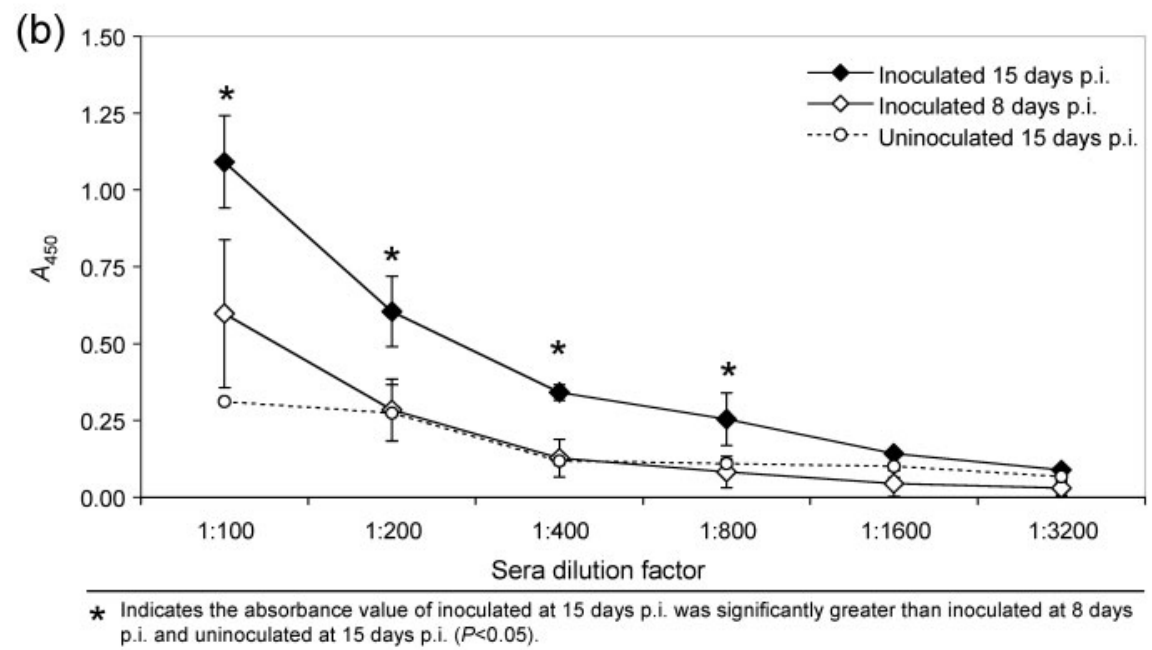

Fig. 3. Anti-C. jejuni antibodies can be detected in the sera of chickens 15 days p.i. with C. jejuni. (a) Caecal samples were collected at 8 and 15 days p.i. from $C$. jejuni-inoculated chickens $(\diamond)$ and from uninoculated chickens $(\bigcirc)$. The c.f.u. per gram of caecal content was determined as described in Methods. (b) Sera were collected at 8 and 15 days p.i. from C. jejuniinoculated and -uninoculated chickens. $\diamond$, Sera from the $C$. jejuni-inoculated chickens at 15 days p.i.; $\diamond$, sera from the $C$. jejuni-inoculated chickens at 8 days p.i.; $\bigcirc$, sera from the uninoculated chickens at 15 days p.i. The ELISAs were performed as described in Methods. chlL- 8 was not detected in the sera of any of the chickens.

compared with human cells. The reduction in C. jejuni invasion of LMH cells was judged to be host-cell-specific because S. typhimurium invaded the LMH and INT 407 cells at comparable levels. To ensure that the low efficiency observed for C. jejuni invasion of LMH cells was not due to the hepatocellular origin of the cells, binding and invasion assays were performed with the human HepG2 hepatocellular cells. All of the C. jejuni strains used in this study bound and invaded the HepG2 cells at levels comparable with the INT 407 cells (results not shown). In contrast to these results, Smith et al. (2005) and Byrne et al. (2007) reported that $C$. jejuni isolates invade chicken primary cells and human cells at comparable levels. Byrne et al. (2007) did find that one $C$. jejuni isolate (i.e. poultry strain $1646 \mathrm{DF})$ invades human cells in greater numbers than it invades chicken cells. Interestingly, Van Deun et al. (2007) reported that $C$. jejuni does not invade chicken embryonic fibroblast cells. Van Deun et al. (2007) also reported that $C$. jejuni invades primary caecal epithelial cells, but at a level that is significantly less than observed with T84 human colonic epithelial cells. The differences reported in C. jejuni invasion of chicken cells suggest that the specific cell type may dictate the invasive phenotype observed rather than the virulence of the specific $C$. jejuni strain, and raises the possibility that the behaviour of LMH cells may not mimic that of cells in vivo. These differences also highlight the need for additional chicken continuous cell lines for comparative studies. In summary, we found that $C$. jejuni invades chicken LMH epithelial cells in significantly lower numbers than it invades human INT 407 epithelial cells. 

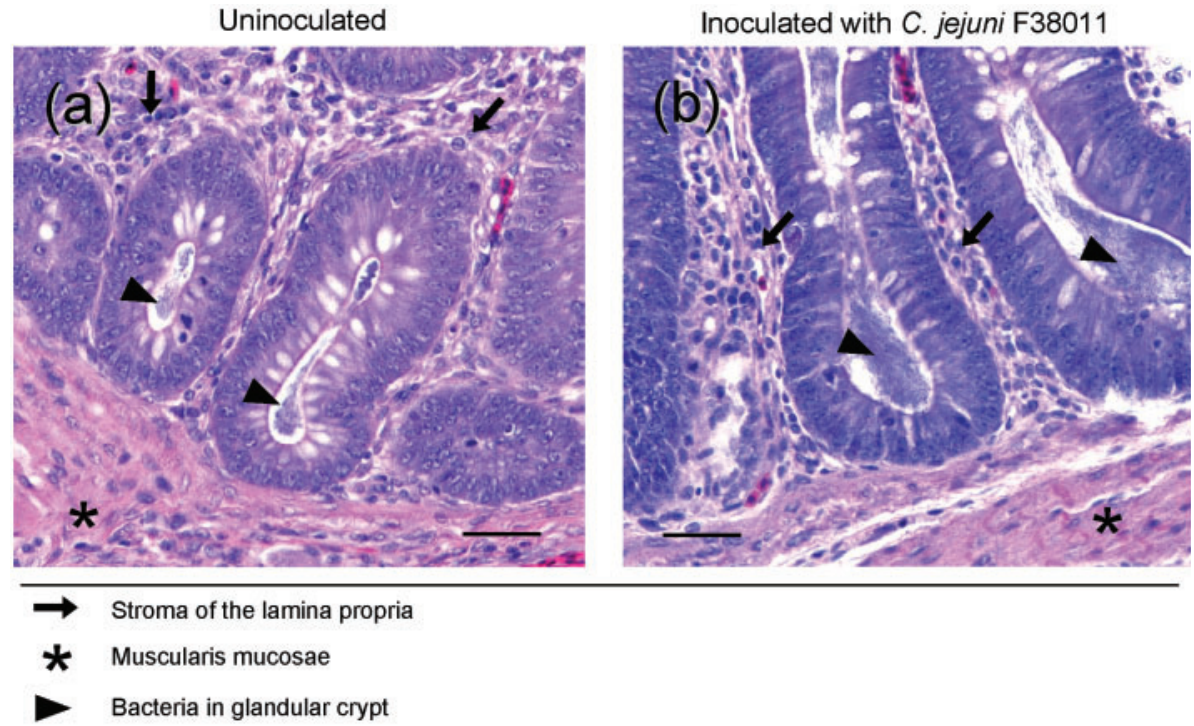

Fig. 4. Deep caecal glands from an uninoculated (a) and C. jejuni F38011-inoculated (b) chicken at 15 days p.i. Note the colonization of the deep mucosal glands by slender rod-shaped bacteria (arrow heads) in both chickens and the lack of any epithelial hyperplastic response or stromal leukocyte infiltration (arrows). The caecal muscularis mucosae is indicated with an asterisk. C. jejuni bacteria were not recovered from the caecal sample of the uninoculated chicken (a), but were recovered from the caecal sample of the C. jejuni F38011-inoculated chicken (b). Both images were acquired at $\times 400$ magnification. Bars, $50 \mu \mathrm{m}$.
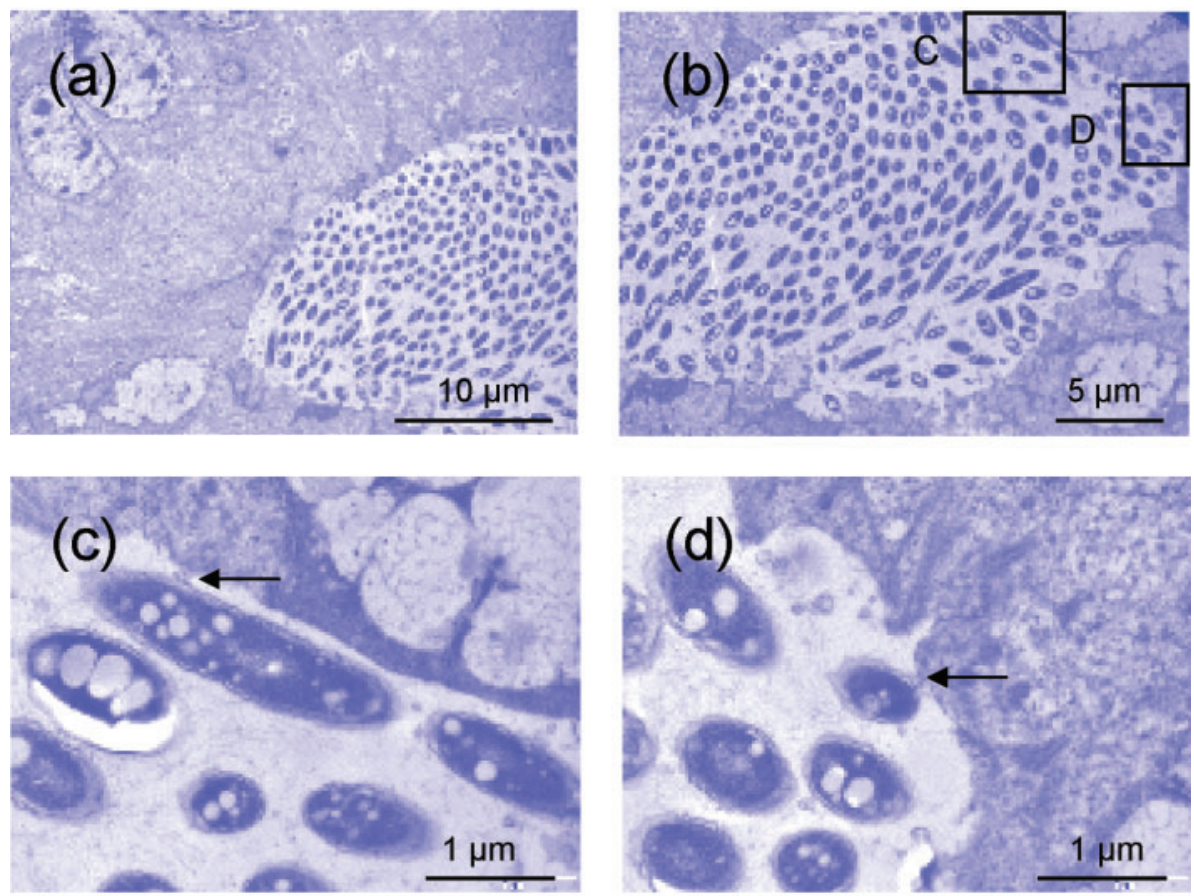

Fig. 5. Transmission electron micrographs of caecal tissues from a C. jejuni F38011-inoculated chicken at 15 days p.i. (a, b) C. jejuni in the glandular crypt. Infiltration of the tissue surrounding the crypt by the bacteria was not apparent. (c, d) Enlarged images of the boxed areas in (b). Contact between C. jejuni and host tissue is indicated by the arrows. 
The transcript levels of chCXCLi2 and chCXCLi1 in LMH cells and of huCXCL8 in INT 407 cells inoculated with $C$. jejuni were assessed by real-time RT-PCR. Each of the four C. jejuni strains induced the expression of both chCXCLi1 and chCXCLi2 from LMH cells. The expression of chCXCLi1 in C. jejuni-inoculated LMH cells was greater than that of chCXCLi2. While this finding is in agreement with the results of Li et al. (2008) with primary chicken embryo intestinal cells, it is in contrast to the findings of Borrmann et al. (2007) and Smith et al. (2005) with primary chicken cells. We also assessed chCXCLil and chCXCLi2 transcript levels in response to $S$. typhimurium as a biological control (Withanage et al., 2004). In contrast to C. jejuni, the expression of chCXCLi2 was greater than that of chCXCLi1 in S. typhimurium-inoculated LMH cells. The relevance of the differences in chCXCLi1 and chCXCLi2 expression from C. jejuni-inoculated LMH cells is not known. While chCXCLi2 is chemotactic for macrophages and lymphocytes (Martins-Green \& Feugate, 1998), the function of chCXCLi1 has been less intensively studied and is not yet defined.

Earlier studies have revealed that $C$. jejuni induces secretion of huCXCL8 from a variety of human epithelial cells, dendritic cells and macrophages (Hickey et al., 1999; Hu et al., 2006; Johanesen \& Dwinell, 2006; MacCallum et al., 2006). Therefore, we performed experiments to determine whether chIL-8 (chCXCLi1 and/or chCXCLi2) is secreted from LMH epithelial cells. chIL-8 was detected in LMH cells inoculated with S. typhimurium, but not in supernatants from LMH cells inoculated with $C$. jejuni, as judged by ELISA. We also found that all four C. jejuni strains induced huCXCL8 secretion from human HepG2 cells (not shown), indicating that the absence of detectable chIL-8 was not an effect resulting from the hepatocellular origin of LMH cells.

Transcriptional, post-transcriptional and post-translational regulatory mechanisms determine whether cytokines are secreted to initiate the inflammatory response (Hamilton et al., 2007). Earlier studies report that cytokine transcript levels are increased in primary chicken cells inoculated with C. jejuni, but these studies did not assess secretion and activity of cytokines (Borrmann et al., 2007; Smith et al., 2005). We evaluated whether chicken cells inoculated with C. jejuni secrete chemoattractants capable of recruiting chicken heterophils. We found that supernatant fluids collected from C. jejuni-inoculated LMH cells induced an increase in chicken heterophil migration relative to the untreated control supernatant fluids. However, the number of heterophils that migrated in response to supernatants from C. jejuni-infected cells was significantly lower than the number of heterophils that migrated in response to $S$. typhimurium-inoculated chicken cells. Based on these assays, we concluded that the response of chicken LMH cells to C. jejuni is distinct from that to $S$. typhimurium, in that $S$. typhimurium induces the production of cytokines that trigger a robust heterophil migration response.
Two in vivo experiments were conducted with broiler chickens inoculated with the C. jejuni F38011 strain to assess colonization, pathology of the caecal mucosa, production of serum antibodies, and serum chIL-8 levels. In the first experiment, we found that $10^{6}$ C. jejuni $\mathrm{F} 38011$ were sufficient for colonization of all chickens at 6 days p.i. In the second experiment, chickens were inoculated with $10^{7}$ C. jejuni F38011, and caecal tissue and serum samples were collected at 8 and 15 days p.i. Anti-Campylobacter antibodies were not detected in the sera of C. jejuniinoculated chickens at 8 days p.i., but both $\operatorname{IgG}$ and $\operatorname{IgM}$ antibodies were present in the serum of the C. jejuniinoculated chickens at 15 days p.i., as judged by immunoblot analysis and ELISAs. More specifically, the level of anti-C. jejuni IgG antibodies detected in colonized chickens at 15 days p.i. was significantly greater than the antibody levels in the C. jejuni-uninoculated chickens at both 8 and 15 days p.i., as well as in the chickens colonized by $C$. jejuni at 8 days p.i. chIL- 8 was not detected in the sera from any of the chickens over the course of the experiment. Collectively, these data demonstrate that antiCampylobacter antibodies are produced in C. jejuniinoculated chickens between 1 and 2 weeks p.i., and that the production of these antibodies occurs in the apparent absence of stimulation of the chIL-8 pro-inflammatory cytokine. While it is possible that chIL-8 secretion occurred at times other than at 8 and 15 days p.i., overt pathology was not observed in the C. jejuni-inoculated chickens (see below). These observations distinguish the immune response of chickens to $C$. jejuni from that of humans. First, humans infected with $C$. jejuni have detectable serum levels of huCXCL8 that peak between 3 and 10 days p.i. (Baqar et al., 2001). Second, the kinetics of antibody production against $C$. jejuni is distinct in chickens compared with humans. In humans, an increase in antiC. jejuni antibodies is detected at approximately 7 days post-infection (Baqar et al., 2001). However, we were unable to detect anti-C. jejuni antibodies in the chickens inoculated with a large dose of C. jejuni F38011 $\left(\sim 10^{7}\right.$ organisms) until 15 days p.i.

Caecal tissues were collected to assess pathology of tissues in chickens harbouring C. jejuni. It was not possible to distinguish the tissue samples of the C. jejuni-inoculated chickens from those of the uninoculated chickens by either haematoxylin and eosin staining or TEM. None of the $C$. jejuni-inoculated chickens exhibited epithelial necrosis or hyperplasia. In addition, we did not observe infiltration of heterophils, lymphocytes or plasma cells into the stroma of the lamina propria. C. jejuni were observed in loose association with the cells lining the caecal gland; areas of bacteria-host-cell binding were observed in which small fibres appeared to serve as a bridge between the cells. $C$. jejuni invasion of the tissue was not observed.

Beery et al. (1988) and Lee et al. (1986) concluded that C. jejuni does not adhere to the gut mucosa of chickens or mice, respectively, and that bacterial adherence is likely not important for the colonization of a host. Although not the 
primary focus of this study, C. jejuni were observed in association with the cells of the caecal gland by TEM. The tissue-associated bacteria were confirmed to be $C$. jejuni by TEM coupled with immunogold labelling using antiCampylobacter-specific antibodies (results not shown). Moreover, studies have been published since the mid1980s demonstrating that specific proteins play a role in, and in some cases are required for, $C$. jejuni colonization of chickens. Two C. jejuni proteins involved in colonization, presumably due to their adhesive properties, are CadF and CapA (Ashgar et al., 2007; Konkel et al., 1997). Ziprin et al. (1999) reported that a C. jejuni cadF mutant is incapable of colonizing chickens. Furthermore, by comparative proteomics, Seal et al. (2007) found that a robust colonizing isolate of $C$. jejuni synthesizes the CadF protein in a greater amount than a poorly colonizing isolate. Additional studies are warranted to dissect the specific interactions of $C$. jejuni with cells lining the glandular crypts.

The data obtained from the ELISA, RT-PCR and heterophil migration experiments raise the concern that the ELISA may not detect both chCXCLi1 and chCXCLi2. As stated above, we found that the inoculation of LMH cells with $C$. jejuni stimulated expression of both $h u C X C L 8$ orthologues, chCXCLi1 and chCXCLi2, but at levels significantly lower than huCXCL8 expressed from human INT 407 cells inoculated with $C$. jejuni. The supernatant fluids of LMH cells co-cultured with $C$. jejuni did not contain detectable amounts of chIL-8, as judged by the sandwich ELISA, but these same supernatant fluids did induce some chicken heterophil migration. Interestingly, chIL-8 was detected from LMH cells inoculated with S. typhimurium using the sandwich ELISA. Taken together, it is not clear whether the capture and detection antibodies reacted against both chCXCLi1 and chCXCLi2. The RT-PCR and heterophil migration data support the finding by ELISA that $S$. typhimurium stimulates chIL-8 secretion, but both assays also indicate that the ELISA has low sensitivity, since chIL-8 was not detected in supernatant fluids from C. jejuniinoculated LMH cells. The detection of chIL-8 in the sera collected from the $C$. jejuni-inoculated broiler chickens was dependent on the sandwich ELISA, and due to the possibility that the assay may not be sensitive enough to detect both chCXCLi1 and chCXCLi2, it is feasible that at least one of these chemokines is produced and participates in inducing the humoral immune response against $C$. jejuni.

In summary, binding and internalization assays revealed that C. jejuni was significantly less invasive for LMH cells relative to human INT 407 cells, whereas S. typhimurium was able to invade both cell types at similar levels. We also found that the expression of chCXCLi2 was greater than that of chCXCLil for S. typhimurium-inoculated LMH cells, but that chCXCLi1 expression was greater than chCXCLi2 expression in LMH cells inoculated with $C$. jejuni. chCXCLi1 and chCXCLi2 have approximately 48 and $50 \%$ amino acid identity to huCXCL8, respectively, and share $67 \%$ identity with one another (Sick et al., 2000). Based on our data, it is possible that either
chCXCLi1 or chCXCLi2 is not recognized by the huCXCL8 capture and detection antibodies. Because the production of chCXCLil from C. jejuni-inoculated LMH cells was similar with $S$. typhimurium-inoculated LMH cells, we hypothesize that the huCXCL8 antibodies used in the ELISA have greater affinity for chCXCLi2 than chCXCLi1. This hypothesis is also supported by the heterophil migration assay, in which S. typhimurium stimulated greater heterophil migration than $C$. jejuni. Noteworthy is that chCXCLi2 has been shown to be chemotactic for monocytes and lymphocytes (Martins-Green \& Feugate, 1998), whereas the chemotactic potential of chCXCLil has not yet been demonstrated. Based on these data, we conclude that chicken LMH cells and human INT 407 cells respond differently to C. jejuni versus S. typhimurium. Additional studies are needed to elucidate the role of chCXCLil and chCXCLi2 in response to C. jejuni.

\section{ACKNOWLEDGEMENTS}

We thank Dr Jeffrey Christensen and Dennis Schaberg for assistance with the inoculation of the chickens with C. jejuni and sample collection, Stuart Perry for animal care, and Sylvia K. Weber and Fonda Wier for assistance in preparing chicken tissue samples for plating. Finally, we thank Dr Phil Mixter and Jason Neal-McKinney for critical review of this manuscript. This work was supported from funds awarded to M.E. K. by the USDA National Research Initiative's Food Safety 32.0 program (2006-35201-17305) and USDA NRI through the Food Safety Research Response Network (2005-3521215287).

\section{REFERENCES}

Allos, B. M. (2001). Campylobacter jejuni infections: update on emerging issues and trends. Clin Infect Dis 32, 1201-1206.

Andreasen, C. B. \& Latimer, K. S. (1989). Separation of avian heterophils from blood using Ficoll-Hypaque discontinuous gradients. Avian Dis 33, 163-167.

Ashgar, S. S., Oldfield, N. J., Wooldridge, K. G., Jones, M. A., Irving, G. J., Turner, D. P. \& Ala'Aldeen, D. A. (2007). CapA, an autotransporter protein of Campylobacter jejuni, mediates association with human epithelial cells and colonization of the chicken gut. $J$ Bacteriol 189, 1856-1865.

Bakhiet, M., Al-Salloom, F. S., Qareiballa, A., Bindayna, K., Farid, I. \& Botta, G. A. (2004). Induction of $\alpha$ and $\beta$ chemokines by intestinal epithelial cells stimulated with Campylobacter jejuni. J Infect 48, 236244.

Baqar, S., Rice, B., Lee, L., Bourgeois, A. L., El Din, A. N., Tribble, D. R., Heresi, G. P., Mourad, A. S. \& Murphy, J. R. (2001). Campylobacter jejuni enteritis. Clin Infect Dis 33, 901-905.

Beery, J. T., Hugdahl, M. B. \& Doyle, M. P. (1988). Colonization of gastrointestinal tracts of chicks by Campylobacter jejuni. Appl Environ Microbiol 54, 2365-2370.

Black, R. E., Levine, M. M., Clements, M. L., Hughes, T. P. \& Blaser, M. J. (1988). Experimental Campylobacter jejuni infection in humans. J Infect Dis 157, 472-479.

Blaser, M. J., Wells, J. G., Feldman, R. A., Pollard, R. A. \& Allen, J. R. (1983). Campylobacter enteritis in the United States. A multicenter study. Ann Intern Med 98, 360-365. 
Blaser, M. J., Black, R. E., Duncan, D. J. \& Am, J. (1985). Campylobacter jejuni-specific serum antibodies are elevated in healthy Bangladeshi children. J Clin Microbiol 21, 164-167.

Blikslager, A. T., Moeser, A. J., Gookin, J. L., Jones, S. L. \& Odle, J. (2007). Restoration of barrier function in injured intestinal mucosa. Physiol Rev 87, 545-564.

Borrmann, E., Berndt, A., Hanel, I. \& Kohler, H. (2007). Campylobacter-induced interleukin-8 responses in human intestinal epithelial cells and primary intestinal chick cells. Vet Microbiol 124, $115-124$

Byrne, C. M., Clyne, M. \& Bourke, B. (2007). Campylobacter jejuni adhere to and invade chicken intestinal epithelial cells in vitro. Microbiology 153, 561-569.

Cawthraw, S., Ayling, R., Nuijten, P., Wassenaar, T. \& Newell, D. G. (1994). Isotype, specificity, and kinetics of systemic and mucosal antibodies to Campylobacter jejuni antigens, including flagellin, during experimental oral infections of chickens. Avian Dis 38, 341349.

Everest, P. H., Goossens, H., Butzler, J. P., Lloyd, D., Knutton, S. Ketley, J. M. \& Williams, P. H. (1992). Differentiated Caco-2 cells as a model for enteric invasion by Campylobacter jejuni and C. coli. J Med Microbiol 37, 319-325.

Fleckenstein, J. M. \& Kopecko, D. J. (2001). Breaching the mucosal barrier by stealth: an emerging pathogenic mechanism for enteroadherent bacterial pathogens. J Clin Invest 107, 27-30.

Hamilton, T. A., Novotny, M., Datta, S., Mandal, P., Hartupee, J., Tebo, J. \& Li, X. (2007). Chemokine and chemoattractant receptor expression: post-transcriptional regulation. J Leukoc Biol 82, 213-219.

Hickey, T. E., Baqar, S., Bourgeois, A. L., Ewing, C. P. \& Guerry, P. (1999). Campylobacter jejuni-stimulated secretion of interleukin- 8 by INT407 cells. Infect Immun 67, 88-93.

Hu, L., Bray, M. D., Osorio, M. \& Kopecko, D. J. (2006). Campylobacter jejuni induces maturation and cytokine production in human dendritic cells. Infect Immun 74, 2697-2705.

Johanesen, P. A. \& Dwinell, M. B. (2006). Flagellin-independent regulation of chemokine host defense in Campylobacter jejuni-infected intestinal epithelium. Infect Immun 74, 3437-3447.

Kaiser, P., Hughes, S. \& Bumstead, N. (1999). The chicken 9E3/CEF4 CXC chemokine is the avian orthologue of IL8 and maps to chicken chromosome 4 syntenic with genes flanking the mammalian chemokine cluster. Immunogenetics 49, 673-684.

Kaiser, P., Rothwell, L., Galyov, E. E., Barrow, P. A., Burnside, J. \& Wigley, P. (2000). Differential cytokine expression in avian cells in response to invasion by Salmonella typhimurium, Salmonella enteritidis and Salmonella gallinarum. Microbiology 146, 3217-3226.

Kelly, D., Conway, S. \& Aminov, R. (2005). Commensal gut bacteria: mechanisms of immune modulation. Trends Immunol 26, 326-333.

Konkel, M. E., Garvis, S. G., Tipton, S. L., Anderson, D. E., Jr \& Cieplak, W., Jr (1997). Identification and molecular cloning of a gene encoding a fibronectin-binding protein ( $\mathrm{CadF})$ from Campylobacter jejuni. Mol Microbiol 24, 953-963.

Konkel, M. E., Monteville, M. R., Rivera-Amill, V. \& Joens, L. A. (2001). The pathogenesis of Campylobacter jejuni-mediated enteritis. Curr Issues Intest Microbiol 2, 55-71.
Lee, A., O'Rourke, J. L., Barrington, P. J. \& Trust, T. J. (1986). Mucus colonization as a determinant of pathogenicity in intestinal infection by Campylobacter jejuni: a mouse cecal model. Infect Immun 51, 536546.

Li, Y.-P., Ingmer, H., Madsen, M. \& Bang, D. D. (2008). Cytokine responses in primary chicken embryo intestinal cells infected with Campylobacter jejuni strains of human and chicken origin and the expression of bacterial virulence-associated genes. BMC Microbiol 8, 107.

MacCallum, A. J., Harris, D., Haddock, G. \& Everest, P. H. (2006). Campylobacter jejuni-infected human epithelial cell lines vary in their ability to secrete interleukin-8 compared to in vitro-infected primary human intestinal tissue. Microbiology 152, 3661-3665.

Martins-Green, M. \& Feugate, J. E. (1998). The 9E3/CEF4 gene product is a chemotactic and angiogenic factor that can initiate the wound-healing cascade in vivo. Cytokine 10, 522-535.

Nathan, C. (2006). Neutrophils and immunity: challenges and opportunities. Nat Rev Immunol 6, 173-182.

Neish, A. S., Gewirtz, A. T., Zeng, H., Young, A. N., Hobert, M. E., Karmali, V., Rao, A. S. \& Madara, J. L. (2000). Prokaryotic regulation of epithelial responses by inhibition of $\mathrm{I} \kappa \mathrm{B}-\alpha$ ubiquitination. Science 289, 1560-1563.

O'Hara, A. M. \& Shanahan, F. (2006). The gut flora as a forgotten organ. EMBO Rep 7, 688-693.

Ramos, H. C., Rumbo, M. \& Sirard, J. C. (2004). Bacterial flagellins: mediators of pathogenicity and host immune responses in mucosa. Trends Microbiol 12, 509-517.

Seal, B. S., Hiett, K. L., Kuntz, R. L., Woolsey, R., Schegg, K. M., Ard, M. \& Stintzi, A. (2007). Proteomic analyses of a robust versus a poor chicken gastrointestinal colonizing isolate of Campylobacter jejuni. J Proteome Res 6, 4582-4591.

Sick, C., Schneider, K., Staeheli, P. \& Weining, K. C. (2000). Novel chicken CXC and CC chemokines. Cytokine 12, 181-186.

Smith, C. K., Kaiser, P., Rothwell, L., Humphrey, T., Barrow, P. A. \& Jones, M. A. (2005). Campylobacter jejuni-induced cytokine responses in avian cells. Infect Immun 73, 2094-2100.

Van Deun, K., Pasmans, F., Ducatelle, R., Flahou, B., Vissenberg, K., Martel, A., Van den Broeck, W., Van Immerseel, F. \& Haesebrouck, F. (2007). Colonization strategy of Campylobacter jejuni results in persistent infection of the chicken gut. Vet Microbiol 130, 285-297.

Wassenaar, T. M. \& Blaser, M. J. (1999). Pathophysiology of Campylobacter jejuni infections of humans. Microbes Infect 1, 10231033.

Withanage, G. S., Kaiser, P., Wigley, P., Powers, C., Mastroeni, P., Brooks, H., Barrow, P., Smith, A., Maskell, D. \& McConnell, I. (2004). Rapid expression of chemokines and proinflammatory cytokines in newly hatched chickens infected with Salmonella enterica serovar Typhimurium. Infect Immun 72, 2152-2159.

Ziprin, R. L., Young, C. R., Stanker, L. H., Hume, M. E. \& Konkel, M. E. (1999). The absence of cecal colonization of chicks by a mutant of Campylobacter jejuni not expressing bacterial fibronectin-binding protein. Avian Dis 43, 586-589.

Edited by: P. H. Everest 